\title{
Cosmic ray acceleration at supergalactic accretion shocks: A new upper energy limit due to a finite shock extension
}

\author{
M. Ostrowski and G. Siemieniec-Oziębło
}

Obserwatorium Astronomiczne, Uniwersytet Jagielloński, ul. Orla 171, 30-244 Kraków, Poland

Received 23 October 2001 / Accepted 18 February 2002

\begin{abstract}
Accretion flows onto supergalactic-scale structures are accompanied with large spatial scale shock waves. These shocks were postulated as possible sources of ultra-high energy cosmic rays. The highest particle energies were expected for perpendicular shock configuration in the so-called "Jokipii diffusion limit", involving weakly turbulent conditions in the large-scale magnetic field imbedded in the accreting plasma. For such configuration we discuss the process limiting the highest energy that particles can obtain in the first-order Fermi acceleration process due to finite shock extensions to the sides, along and across the mean magnetic field. Cosmic ray outflow along the shock structure can substantially lower the upper energy limit for conditions considered for supergalactic shocks.
\end{abstract}

Key words. ISM: cosmic rays - acceleration of particles - shock waves - galaxies: clusters: general

\section{Introduction}

Formation of large-scale extragalactic structures (galaxy clusters and super-clusters) involving accretion of plasma to the gravitational potential wells is accompanied by formation of strong shock waves (Ryu et al. 1993). These shocks were considered as viable sources of ultra high energy cosmic rays (UHECRs), up to energies $E_{\max } \sim$ $10^{18-19} \mathrm{eV}$ (Kang et al. 1997; Hillas 1984). Evaluation of this maximum energy is not reliable due to inadequate knowledge of physical conditions at the shocks considered. The first estimates show that maximal particle energies accelerated in such conditions must be limited because of the extensive time scales involved. The magnetic fields in the considered flows are not expected to be stronger than $\sim 1 \mu \mathrm{G}$ for the accretion in rich galaxy clusters and $\sim 0.1 \mu \mathrm{G}$ for supergalaxy scale accretion (Kronberg 2001). At the same time the former flows can involve plasma velocities $\sim 1000 \mathrm{kms}^{-1}$, while the latter a few times lower ones (Juszkiewicz et al. 2000). The ordinary diffusive Fermi acceleration considered (Drury 1983) is fastest for highly turbulent conditions with the diffusion coefficient comparable to the Bohm diffusion coefficient, $\kappa_{\mathrm{B}}$. In the considered situations, the involved acceleration time scale $\tau \sim \kappa_{\mathrm{B}} / V_{\text {shock }}^{2}$ becomes longer than the age of the universe for particle energies greater than $\sim 10^{18} \mathrm{eV}$.

However, as pointed out by Kang et al. (1997; cf. Jokipii 1987; Ostrowski 1988), even faster acceleration can

Send offprint requests to: G. Siemieniec-Oziȩbło, e-mail: grazyna@oa.uj.edu.pl take place at perpendicular shocks occurring when largescale magnetic field structures are present in the intergalactic medium and the accretion proceeds perpendicular to the field direction. Then, the cosmic ray cross-field diffusion coefficient governs the first order Fermi acceleration process at the shock. With the involved spatial scales along the shock normal of respectively $1 \mathrm{Mpc}$ for a cluster, or a few Mpc for a super-cluster flow, there is enough space and time to accelerate particles up to $\sim 10^{21} \mathrm{eV}$, three orders of magnitude greater than in the Bohm diffusion limit (cf. Siemieniec-Oziębło \& Ostrowski 2000).

Below, in Sect. 2, we present evaluations of the acceleration time scales in perpendicular shocks. The derived time scale is used in Sect. 3 to discuss the particle diffusion and drifts along the shock front, perpendicular to the plasma flow direction. We show that particle escape to the sides of the shock structure may substantially limit $E_{\max }$ derived for perpendicular shocks. In Sect. 4 we briefly discuss the importance of this constraint for large-scale extragalactic shocks.

\section{Particle acceleration at perpendicular shock waves}

The acceleration time scale derived within the diffusion approximation for oblique shocks (Ostrowski 1988) becomes formally invalid for perpendicular shocks which are always superluminal. Nevertheless, Jokipii (1987) used the 


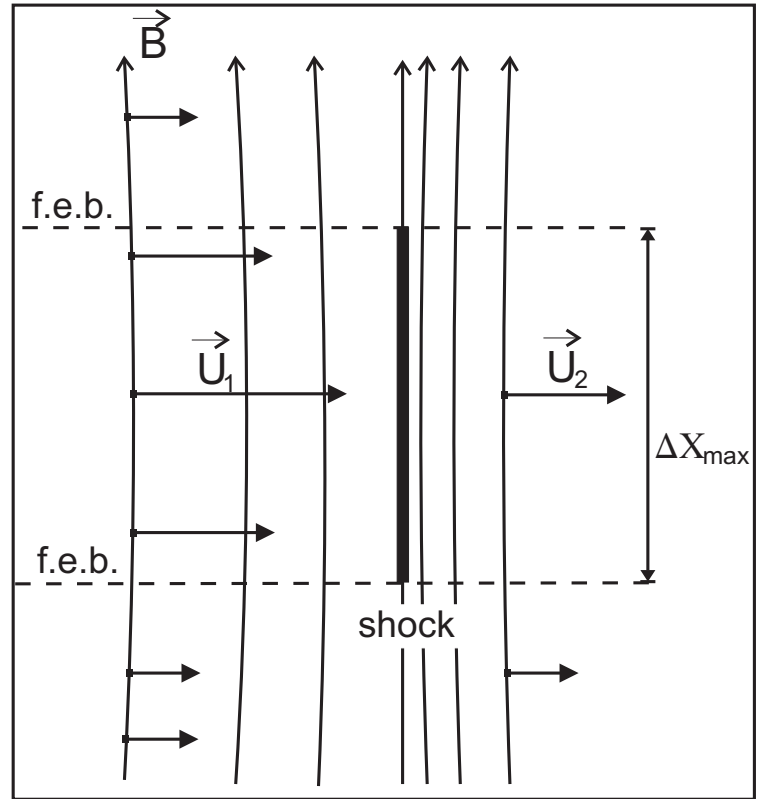

Fig. 1. Qualitative sketch of the physical situation considered near the extragalactic-scale accretion shock: lines represent the mean magnetic field which is (quasi) perpendicular to the plasma flow at the shock. A non-uniformity of the flow results in a finite shock extension along the mean magnetic field $\Delta X_{\max }$. Particle escape along the magnetic field from the acceleration region at the shock is represented by the introduced free escape boundaries ("f.e.b.") indicated by dashed lines).

diffusion equation to derive the energetic particle acceleration time scale for such shocks:

$\tau=\frac{3}{U_{1}-U_{2}}\left(\frac{\kappa_{1}}{U_{1}}+\frac{\kappa_{2}}{U_{2}}\right)$,

where $\kappa_{1}$ and $U_{1}\left(\kappa_{2}\right.$ and $\left.U_{2}\right)$ are the normal diffusion coefficient and shock velocity as measured upstream (downstream) of the shock. With the scaling assumed by Jokipii (1987)

$\frac{\kappa_{\perp}}{\kappa_{\|}}=\frac{1}{1+\left(\frac{\lambda_{\|}}{r_{\mathrm{g}}}\right)^{2}}$,

where symbols $\perp$ and $\|$ indicate quantities perpendicular and parallel to the mean magnetic field, respectively, and $\lambda_{\|}$is a mean free path for particle scattering along the magnetic field $\left(\kappa_{\|}=\frac{1}{3} \lambda_{\|} w\right.$, where $w$ is a particle velocity), and $r_{\mathrm{g}}=p c / e B$ is the particle gyroradius. The Eq. (2.2) describes the cross field diffusion, where, at the mean free time, the particle moves on average $\lambda_{\|}$along the magnetic field and is scattered at a distance $r_{\mathrm{g}}$ across it. Continuing the acceleration process at perpendicular shocks requires sufficiently effective particle cross-field diffusion. With $\eta \equiv \lambda_{\|} / r_{\mathrm{g}}$ this requirement can be expressed (Jokipii 1987) as

$\eta<\frac{w}{U_{1}}$

\section{Maximum energy of accelerated particles}

\section{1. $\tau$ : A diffusive approach}

In order to evaluate the maximum energy attainable in the acceleration process acting at the perpendicular shock wave of a finite extension $\Delta X_{\max }$ along the magnetic field one has to consider particle escape from the shock region, represented by free escape boundaries of Fig. 1. Let us assume Eq. (2.1) to be a valid acceleration time estimate. Additionally, we consider conditions with the constant $\eta$ upstream and downstream of the shock i.e. $\eta=\lambda_{\|, 1} / r_{\mathrm{g}, 1}=$ $\lambda_{\|, 2} / r_{\mathrm{g}, 2}$. With the shock compression ratio $r \equiv U_{1} / U_{2}$ and a weakly perturbed upstream magnetic field $B_{1}$, the downstream magnetic field $B_{2}=r B_{1}$ and the particle gyroradius becomes $r_{\mathrm{g}, 2}=r_{\mathrm{g}, 1} / r$. Fast acceleration at perpendicular shocks appears only for weakly turbulent conditions with $\lambda_{\|} \gg r_{\mathrm{g}}$. From Eq. (2.2), in this limit $\kappa_{\perp}=\kappa_{\|}\left(\frac{r_{g}}{\lambda_{\|}}\right)^{2}$. For simplicity let us consider a purely perpendicular shock with the mean magnetic field parallel to the shock surface and, respectively, $\kappa_{1}=\kappa_{\perp, 1}$ and $\kappa_{2}=\kappa_{\perp, 2}$, and relativistic cosmic ray particles with $w=c$. Then, with the Bohm diffusion coefficient $\kappa_{\mathrm{B}} \equiv \frac{1}{3} r_{\mathrm{g}} c$, the diffusion coefficients

$\kappa_{i}=\frac{1}{\eta} \kappa_{\mathrm{B}, i} \quad(i=1,2)$,

and the Eq. (2.1) transforms to

$\tau=\frac{2 r}{r-1} \frac{1}{\eta} \frac{r_{\mathrm{g}, 1}}{c}\left(\frac{c}{U_{1}}\right)^{2}$.

With the constraint (2.3) and the strong shock with $r=4$ the acceleration time scale must be longer than $c / U_{1}$ times a particle gyroperiod.

\section{2. $\tau$ : A lower limit for the perpendicular shock}

An independent lower limit for the acceleration time scale can be derived by considering physical conditions approaching a weak scattering limit. Then, in the absence of scattering, let us consider the shock-drift acceleration process of upstream particles at the perpendicular shock propagating in a uniform magnetic field. A particle with the perpendicular momentum component $p_{\perp}$ compressed in the shock will increase it to

$p_{\perp, 2}=p_{\perp, 1} \sqrt{r}$

due to the approximate magnetic momentum conservation. For strong shocks, on average particles roughly double their momenta at individual transmissions through the shock, which take approximately

$\Delta t=\frac{2 r_{\mathrm{g}, 1}}{U_{1}}=\frac{2 r_{\mathrm{g}, 2}}{U_{2}}$.

Thus the characteristic acceleration time cannot be shorter than $\tau \sim \Delta t$. The same result can be obtained by considering particle drift along the shock, perpendicular to the weakly perturbed magnetic field. Addition of 
the diffusive process that is required to allow downstream particles to return to the shock to continue acceleration can only make $\tau$ longer. One may note that the minimum acceleration time in the Jokipii (1987) derivation approximately coincides with the above one. Thus, the time (3.4) can be considered to be an absolute lower limit for the acceleration time in the perpendicular shock and thus it defines the ultimate limit for $\kappa_{\perp}$ reduction (or the upper limit for the considered $\eta$ ) in derivation of the expression (3.2).

\subsection{New constraints for $E_{\max }$}

Cosmic ray removal from the acceleration region near the shock proceeds due to particle advection with the plasma flow downstream of the shock and due to diffusion or drifts towards the escape boundaries around the shock region. In the present paper we discuss the role of such escape in limiting the maximum energy of accelerated particles.

\subsubsection{A limit due to escape along the magnetic field}

First let us discuss the diffusive escape along the magnetic field (cf. Fig. 1) limiting the maximum particle energy at the shock of a finite extension. To evaluate this limit we consider a solution of the 1-D spatial diffusion equation for particles moving along the magnetic field (along the $x$-axis of our coordinate frame), with the diffusion coefficient $\kappa_{\|}$. For this evaluation we neglect particle transport perpendicular to this field, both along the shock normal and to the sides. For $N_{\mathrm{o}}$ particles injected at $x=0$ and $t=0$, their distribution along the $x$-axis is given by

$n(x, t)=\frac{N_{\mathrm{o}}}{\sqrt{4 \pi \kappa_{\|} t}} \exp \left[-\frac{x^{2}}{4 \kappa_{\|} t}\right]$.

If a dispersion of this distribution $\sqrt{2 \kappa_{\|} t}$ becomes larger than the shock sideway extension $\Delta X_{\max }$, the accelerated particles tend to efficiently escape to the sides, leading to the spectrum steepening and, finally, to formation of the energy cut-off. Such modification of the particle spectrum is possible when the time required for escape becomes comparable to the acceleration time scale $\tau$, i.e. when the particle dispersion along the $x$-axis over time $\tau$ becomes comparable to the shock extension $\Delta X_{\max }$ :

$\Delta X_{\max } \approx 2 \sqrt{2 \kappa_{\|} \tau}$.

Applying the above Eq. (3.2) and the expression for $\kappa_{\|}$, we obtain the requirement for diffusively accelerated particles at such finite-extent shocks as

$r_{\mathrm{g}} \leq \frac{\Delta X_{\max }}{2} \frac{U}{c}$

As the particle gyroradius $r_{\mathrm{g}}=p c / e B$, this expression provides the upper energy limit for accelerated particles due to particle escape along the mean magnetic field. For nonrelativistic shocks with the factor $U / c \ll 1$, Eq. (3.7) provides a severe constraint for the maximum accelerated particle energy.

\subsubsection{A limit due to drift across the magnetic field}

As discussed in Sect. 3.2, particle acceleration at the perpendicular shock with the weakly perturbed magnetic field involves particle drift along the shock surface, across the magnetic field. Thus, the shock extension along this direction (along the $y$-axis of our coordinate frame) also provides a limit for the maximum energy of accelerated particles. The energy gain of a particle drifting along the shock is

$\Delta E=|q \mathcal{E} L|$

where the " $V \times B$ " electric field $\mathcal{E}=-\left(\boldsymbol{U}_{1} \times \boldsymbol{B}_{1}\right) / c=$ $-\left(\boldsymbol{U}_{2} \times \boldsymbol{B}_{2}\right) / c, q$ is the particle electric charge and $L$ is the distance the particle drifted along the shock $(\Delta E>0)$. With the above expressions for the perpendicular shock the Eq. (3.8) can be rewritten as

$\frac{\Delta E}{E} \approx \frac{U}{c} \frac{L}{r_{\mathrm{g}}}$

In order to double the original energy in this process, $\Delta E=E$, an average particle has to move a distance $L=(c / U) \cdot r_{\mathrm{g}}$ along the shock surface. Thus, if the shock extension available for drifting particles $L=\Delta Y_{\max } / 2$, the maximum energy of particles accelerated by the shock drift mechanism is provided by the condition

$r_{\mathrm{g}} \leq \frac{\Delta Y_{\max }}{2} \frac{U}{c}$

analogous to the above condition (3.7).

\section{Conclusions}

The maximum energy a particle can gain in the drift - diffusion acceleration expressed by the famous Hillas (1984) formula needs more specification concerning the characteristic spatial scales of the shock. Apart from the shock normal extension (Bhattacharjee \& Sigl 2000) one also needs information about its sideway extent $\left(\Delta X_{\max }\right.$ and $\left.\Delta Y_{\max }\right)$ influencing the maximum energy and, at highest energies, the spectral shape of the accelerated particles. As an illustration let us consider an accretion flow with velocity $U=300 \mathrm{~km} \mathrm{~s}^{-1}$, the size of $h \approx 3 \mathrm{Mpc}$ along the flow and of $L \approx 10 \mathrm{Mpc}$ to the sides, harbouring a shock wave inside with, say, $\Delta X_{\max }$ and/or $\Delta Y_{\max } \approx L$. In the perpendicular shock configuration the plasma normal extent provides the upper limit for particle energy expressed as $r_{\mathrm{g}}<h$. On the other hand particle escape to the sides provide the limits (3.7) and (3.10). If the magnetic field in the plasma equals $0.1 \mu \mathrm{G}$, the former constraint requires approximately that $E_{\max }<10^{20} \mathrm{eV}$, while the others provide the limit $E_{\max } \sim 10^{17} \mathrm{eV}$. When varying the above accretion flow parameters within the reasonable ranges one finds that the upper energy limit for particles accelerated at such shocks can hardly exceed a scale of EeV, and in most cases is close to $10^{17} \mathrm{eV}$. This low $E_{\max }$ results from the fact that for the weakly perturbed magnetic field 
required for fast acceleration at the considered perpendicular shock, the accelerated particles can diffuse and drift far along the finite shock to escape to its sides. For highly turbulent conditions and/or the parallel shock configuration a similar value for $E_{\max }$ arises due to the slow acceleration constrained by radiation losses (cf. Kang et al. 1997).

One should note that obtaining the same expressions for constraints (3.7) and (3.10) is not a coincidence. The upper limit (3.7) can be rewritten as $\lambda_{\|} \leq \Delta X_{\max } / 2$, while the limit (3.10) means $L \leq \Delta Y_{\max } / 2$. So, for the highest energy particles their dispersion along the shock can be at most comparable to their mean free path or their maximum drift length. Such particles stream along the magnetic field nearly free, advancing their positions a distance $\sim r_{\mathrm{g}}$ in each gyroperiod, and they drift across the magnetic field changing their $y$ coordinate $\sim r_{\mathrm{g}}$ at each gyroperiod, as well, provided $B_{2} \gg B_{1}$. Thus for a given minimum acceleration time (3.2) or (3.4) such highest energy particles can move comparable distances along and across the magnetic field at the shock.
Acknowledgements. We are grateful to the anonymous referee for useful suggestions. The work was supported by the Komitet Badań Naukowych through the grant PB 258/P03/99/17 (MO) and within the project 2 P03B 11217 (GSO).

\section{References}

Bhattacharjee, P., \& Sigl, G. 2000, Phys. Rep., 327, 109

Drury, L. O' C. 1983, Rep. Prog. Phys., 46, 973

Hillas, A. M. 1984, ARA\&A, 22, 425

Jokipii, J. R. 1987, ApJ, 313, 842

Juszkiewicz, R., Ferreira, P., Feldman, H., Jaffe, A., \& Davis, M. 2000, Science, 287, 109

Kang, H., Rachen, J. P., \& Biermann, P. L. 1997, MNRAS, 286, 257

Kronberg, P. 2001, in High Energy Gamma-Ray Astronomy, ed. F. A. Aharonian, \& H. Völk, AIP Conf. Ser.

Lagage, P. O., \& Cesarsky, C. 1983, A\&A, 125, 249

Ostrowski, M. 1988, MNRAS, 233, 257

Ryu, D., Ostriker, J., Kang, H., \& Cen, R. 1993, ApJ, 414, 1

Siemieniec-Oziębło, G., \& Ostrowski, M. 2000, A\&A, 355, 51 